\title{
Trends in characteristics of surgically treated early gastric cancer patients after the introduction of gastric cancer treatment guidelines in Japan
}

\author{
Norimitsu Tanaka ${ }^{1}$, Hitoshi Katai ${ }^{1}$, Hirokazu Taniguchi ${ }^{2}$, Makoto Saka ${ }^{1}$, Shinji Morita ${ }^{1}$, Takeo Fukagawa $^{1}$, \\ and TAKUJI GOTODA ${ }^{3}$ \\ ${ }^{1}$ Gastric Surgery Division, National Cancer Center Hospital, 5-1-1 Tsukiji, Chuo-ku, Tokyo 104-0045, Japan \\ ${ }^{2}$ Clinical Laboratory Division, National Cancer Center Hospital, Tokyo, Japan \\ ${ }^{3}$ Endoscopy Division, National Cancer Center Hospital, Tokyo, Japan
}

\begin{abstract}
Background. The gastric cancer treatment guidelines (Guidelines) of the Japanese Gastric Cancer Association allow endoscopic treatment and a modified gastrectomy for the treatment of early gastric cancer (EGC). Endoscopic treatment is indicated for EGC with a minimal chance of nodal metastasis. Consequently, surgeons will likely treat an increasing number of EGC patients with greater chance of nodal metastasis using a reduced extent of lymphadenectomy. The aim of this study was to investigate the trends in characteristics and long-term oncological outcomes of surgically treated EGC patients after the introduction of the Guidelines.

Methods. Between 2001 and 2003, 696 patients underwent a gastrectomy according to the Guidelines. These 696 patients (the Guidelines group) were retrospectively compared with 635 patients (the control group) who had undergone a gastrectomy between 1991 and 1995 (before the introduction of the Guidelines).

Results. The incidence of nodal metastasis in mucosal cancers was higher in the Guidelines group than in the control group $(6.5 \%$ vs $2.6 \%)$. The proportion of D2 or greater extended lymphadenectomy in the Guidelines group was lower than that in the control group $(29.7 \%$ vs $62.5 \%)$. Nevertheless, the 5-year survival rate in the Guidelines group was similar to that in the control group $(94.2 \%$ vs $92.3 \%)$.

Conclusion. Surgeons treated more cases of mucosal cancer with nodal metastasis after the introduction of the Guidelines. The long-term oncological outcomes for patients with EGC remained excellent. So far, the Guidelines for the treatment of EGC appear acceptable.
\end{abstract}

Key words Early gastric cancer - Lymph node metastasis . Gastric cancer treatment guidelines

Offprint requests to: $\mathrm{H}$. Katai

Received: September 18, 2009 / Accepted: December 2, 2009

\section{Introduction}

Early gastric cancer (EGC) is defined as invasion confined to the mucosa or submucosa, regardless of the presence of regional lymph node metastasis. In recent years, the incidence of EGC has reached more than $50 \%$ of all gastric cancer cases in Japan. Gastrectomy with D2 lymphadenectomy had been firmly accepted as a standard treatment for every stage of gastric cancer since the 1980s [1,2]. EGC treated with radical surgery has an excellent survival rate, with 5-year survival rates of more than $90 \%$ being reported by both Western [3] and Japanese [4] investigators. However, ever since the clinicopathological features of EGC, such as the incidence of lymph node metastasis, were clarified in the late 1990s, gastrectomy with D2 lymphadenectomy for all patients with EGC has come to be considered as an overtreatment [5-8].

The gastric cancer treatment guidelines (Guidelines) were issued by the Japanese Gastric Cancer Association (JGCA) in March 2001 [9, 10]. The Guidelines were designed to provide standard indications for the selection of treatments for gastric cancer according to the clinical stages of the disease (JGCA classification). The Guidelines allowed endoscopic resection and a modified gastrectomy as treatment modalities for EGC, in addition to a standard gastrectomy. Endoscopic treatment can be utilized in patients with a minimal chance of lymph node metastasis. A modified gastrectomy, including limited lymphadenectomy, can be utilized in patients with EGC beyond the inclusion criteria for endoscopic treatment. Because patients with a minimal chance of lymph node metastasis were excluded as candidates for a gastrectomy, Japanese surgeons are interested in whether surgeons are actually facing an increasing number of EGC cases with a greater chance of lymph node metastasis. Furthermore, whether a modified gastrectomy with a reduced extent 
of lymphadenectomy is sufficient treatment for these cases of EGC with a greater chance of lymph node metastasis is also a topic of interest. We retrospectively investigated the clinicopathological characteristics and long-term oncological outcomes of EGC patients who underwent a gastrectomy after the introduction of the Guidelines and compared our results with those obtained in patients treated before the introduction of the Guidelines.

\section{Patients and methods}

\section{Patients}

Between January 1991 and December 2003, 2218 patients with EGC underwent a gastrectomy with curative intent at the National Cancer Center Hospital, Tokyo. Between March 2001 and December 2003, 696 of these patients underwent a gastrectomy according to the Guidelines. These 696 patients (the Guidelines group) were retrospectively compared with 635 patients (the control group) who had undergone a gastrectomy between January 1991 and December 1995 (before the introduction of the Guidelines). Patients who had undergone a gastrectomy between January 1996 and February 2001 were excluded from this analysis because endoscopic resection and a modified gastrectomy for EGC were frequently performed as investigational treatments during this period. Surgical specimens were examined and scored according to the Japanese classification of gastric carcinoma [9]. The vital statistics for all the patients were obtained from the city registry office and the follow-up records. All the patients were followed up for at least 5 years.

\section{Surgical procedures}

Before the introduction of the Guidelines, a gastrectomy with D2 lymphadenectomy was performed as a standard treatment for every stage of gastric cancer. After the introduction of the Guidelines, definite recommendations for endoscopic treatment were confined to tumors in the mucosal layer; type I, IIa, or depressed type IIc with no ulcers; well- or moderately differentiated adenocarcinoma; and tumors smaller than $2 \mathrm{~cm}$. A modified gastrectomy was utilized in patients with cT1N0 (stage IA) tumors and cT1N1 (stage IB) tumors less than $2.0 \mathrm{~cm}$ in size, excluding patients who fulfilled the criteria for endoscopic resection. The modified gastrectomy allowed a reduced extent of lymphadenectomy, compared with a D2 lymphadenectomy. While the N1 nodes were completely removed, some suprapancreatic N2 nodes, such as the node along the splenic artery, were excluded from the dissection.

\section{Statistical analysis}

Statistical analysis was performed using SPSS for Windows version 17.0 (SPSS, Chicago, IL, USA). The significance of the differences in the patients' clinicopathological features and the incidence of lymph node metastasis were determined using the $\chi^{2}$ test and the Mann-Whitney $U$-test, as appropriate. A 5\% significance level $(P<0.05)$ was considered statistically significant. The survival rate was calculated by the Kaplan-Meier method with $95 \%$ confidence intervals (CIs), and the hazard ratio was calculated by the Cox proportional hazards model with $95 \%$ CIs.

\section{Results}

\section{Patient characteristics}

The male-to-female ratio in the Guidelines group (1.92:1) was significantly lower than that in the control group (2.55: 1; Table 1). The median age in the Guidelines group (62 years) was significantly higher than that in the control group (60 years). There was no difference in the distribution of tumor location. The median tumor size in the Guidelines group $(30.0 \mathrm{~mm})$ was significantly larger than that in the control group $(26.0 \mathrm{~mm})$. The proportion of undifferentiated-type lesions in the Guidelines group (58.0\%) was significantly higher than that in the control group (41.7\%).

The incidence of lymph node metastasis in patients with mucosal cancers in the Guidelines group $(6.5 \%)$ was significantly higher than that in the control group (2.6\%; Table 2). No difference in the incidence of lymph node metastasis in patients with submucosal cancer was noted between the two groups. The proportion of D2 or greater extended lymphadenectomy in the Guidelines group (207/696; 29.7\%) was significantly lower than that in the control group $(397 / 635 ; 62.5 \%)$.

\section{Survival}

The median follow-up period in the Guidelines group was 5.5 years, while that in the control group was 7.5 years. During a 5-year follow-up period, 40 patients $(5.7 \%)$ in the Guidelines group died; 9 of these patients $(1.3 \%)$ died from recurrence. Thirty-eight patients in the control group $(6.0 \%)$ died; 6 of these patients $(0.9 \%)$ died from recurrence. The overall 5-year survival rates were $94.2 \%$ (95\% CI, 92.4\%-96.0\%) in the Guidelines group and $92.3 \%$ (95\% CI, 90.1\%-94.5\%) in the control group. If the relative risk for death was set at one for patients in the control group, the hazard ratio in the Guidelines group was 0.78 (95\% CI, 0.53-1.15) for overall patients. The overall 5-year survival rates for the patients with mucosal cancer were 95.1\% (95\% CI, 
Table 1. Clinicopathological characteristics of patients

\begin{tabular}{lccc}
\hline & $\begin{array}{c}\text { Guidelines } \\
(n=696)\end{array}$ & $\begin{array}{c}\text { Controls } \\
(n=635)\end{array}$ & $P$ value \\
\hline Sex & & & \\
$\quad$ Male/female ratio & 1.92 & 2.55 & 0.018 \\
Age (years) & & & 0.005 \\
$\quad$ Median & 62 & $21-86$ & 0.315 \\
$\quad$ Range & $31-89$ & $69(10.9 \%)$ & \\
Tumor location & $88(12.6 \%)$ & $566(89.1 \%)$ & 0.05 \\
$\quad$ Upper third & $608(87.4 \%)$ & 26.0 & \\
$\quad$ Middle and lower thirds & 30.0 & $2-250$ & 0.121 \\
Tumor size (mm) & $3-186$ & $307(48.3 \%)$ & \\
$\quad$ Median & $307(44.1 \%)$ & $328(51.7 \%)$ & 0.203 \\
$\quad$ Range & $389(55.9 \%)$ & $126(19.8 \%)$ & \\
Depth of invasion & $112(16.1 \%)$ & $10(1.6 \%)$ & \\
$\quad$ Mucosa & $11(1.6 \%)$ & $499(78.6 \%)$ & $<0.001$ \\
$\quad$ Mubmucosa & $573(82.3 \%)$ & $370(58.3 \%)$ & \\
$\quad$ Elevated & $292(42.0 \%)$ & $265(41.7 \%)$ & \\
$\quad$ Flat & Depressed & & \\
Histological type & Differentiated & &
\end{tabular}

Guidelines, gastric cancer treatment guidelines of the Japanese Gastric Cancer Association

${ }^{\text {a }}$ According to the Japanese classification of gastric carcinoma [9]

Differentiated type includes papillary adenocarcinoma and tubular adenocarcinoma. Undifferentiated type includes poorly differentiated adenocarcinoma, signet-ring cell carcinoma, and mucinous adenocarcinoma

Table 2. Incidence of lymph node metastasis according to depth of invasion in the Guidelines and control groups

\begin{tabular}{lccc}
\hline & Guidelines & Controls & $P$ value \\
\hline Depth of invasion & & & \\
Mucosa & $20 / 307(6.5 \%)$ & $8 / 307(2.6 \%)$ & 0.02 \\
Submucosa & $78 / 389(20.1 \%)$ & $70 / 328(21.3 \%)$ & 0.671 \\
Overall & $98 / 696(14.1 \%)$ & $78 / 635(12.3 \%)$ & 0.334
\end{tabular}

Guidelines, gastric cancer treatment guidelines of the Japanese Gastric Cancer Association

92.8\%-97.5\%) in the Guidelines group and 93.7\% (95\% CI, $91.0 \%-96.4 \%)$ in the control group. The hazard ratio in the Guidelines group was 0.83 (95\% CI, 0.441.58 ) for patients with mucosal cancer. The overall 5-year survival rates for the patients with submucosal cancer were $93.5 \%(95 \% \mathrm{CI}, 91.0 \%-96.0 \%)$ in the Guidelines group and 91.0\% (95\% CI, 87.9\%-94.1\%) in the control group. The hazard ratio in the Guidelines group was 0.74 (95\% CI, 0.46-1.19) for patients with submucosal cancer.

\section{Discussion}

The Guidelines allow endoscopic treatment and a modified gastrectomy for EGC. Candidates for endoscopic treatment include patients with small differentiatedtype mucosal cancers. When these tumors were excluded from the indications for gastrectomy, the tumor size in the Guidelines group was larger and the proportion of undifferentiated-type lesions was greater in the Guidelines group, compared with these parameters in the control group, as expected.

The male-to-female ratio was significantly lower in the Guidelines group than in the control group. Differentiated-type carcinomas are more common among men than among women [11]. Because male patients tend to fulfill the criteria for endoscopic resection more commonly than female patients, more men may have undergone endoscopic treatment. The median age was also slightly higher in the Guidelines group. Considering that differentiated-type tumors, which are occasionally treated using endoscopy, are more common among elderly patients $[12,13]$, a lower median age in the Guidelines group would not be unexpected. However, the opposite result was obtained. This result might 
reflect an increase in the number of elderly patients themselves as a result of the prolonged life expectancy in Japan.

The incidence of lymph node metastasis in mucosal cancers was reported to be $1.2 \%-3.3 \%$ [6, 14-17], and all of these patients with EGC underwent a standard gastrectomy. In the present study, the incidence of lymph node metastasis in mucosal cancers in the control group was similar to those study findings $(2.6 \%)$, while that in the Guidelines group was significantly higher $(6.5 \%)$. The reason for this difference is easily interpreted. After the introduction of the Guidelines, endoscopic resection was established as the standard therapy for EGC in patients with a negligible risk of lymph node metastasis; therefore, the incidence of surgically treated patients with lymph node metastasis increased.

The Guidelines recommend D2 gastrectomy only in selected patients. As a result, the proportion of patients undergoing gastrectomy with $\mathrm{D} 2$ or greater extended lymphadenectomy was significantly smaller in our Guidelines group (29.7\%) than in the control group $(62.5 \%)$. Nevertheless the 5-year survival rate in the Guidelines group $(94.2 \%)$ was similar to that in the control group $(92.3 \%)$ and was also similar to that for patients undergoing gastrectomy with D2 lymphadenectomy in previous reports $[18,19]$.

In conclusion, surgeons treated more cases of mucosal cancer with lymph node metastasis after the introduction of the Guidelines. The long-term oncological outcomes for patients with EGC remained excellent after the introduction of the modified operation. So far, the Guidelines for the treatment of EGC appear acceptable.

\section{References}

1. Maruyama K, Okabayashi K, Kinoshita T. Progress in gastric cancer surgery in Japan and its limits of radicality. World J Surg 1987;11:418-25.

2. Sawai K, Takahashi T, Suzuki H. New trends in surgery for gastric cancer in Japan. J Surg Oncol 1994;56:221-6.

3. Oliveira FJ, Ferrao H, Furtado E, Batista H, Conceicao L. Early gastric cancer: report of 58 cases. Gastric Cancer 1998;1:51-6.
4. Katai H, Yoshimura K, Maruyama K, Sasako M, Sano T. Evaluation of the New International Union Against Cancer TNM staging for gastric carcinoma. Cancer 2000;88:1796-800.

5. Gotoda T, Yanagisawa A, Sasako M, Ono H, Nakanishi Y, Shimoda $\mathrm{T}$, et al. Incidence of lymph node metastasis from early gastric cancer: estimation with a large number of cases at two large centers. Gastric Cancer 2000;3:219-25.

6. Sano T, Kobori O, Muto T. Lymph node metastasis from early gastric cancer: endoscopic resection of tumour. Br J Surg 1992; 79:241-4.

7. Yamao T, Shirao K, Ono H, Kondo H, Saito D, Yamaguchi H, et al. Risk factors for lymph node metastasis from intramucosal gastric carcinoma. Cancer 1996;77:602-6.

8. Saka M, Katai H, Fukagawa T, Nijjar R, Sano T. Recurrence in early gastric cancer with lymph node metastasis. Gastric Cancer 2008;11:214-8.

9. Japanese Gastric Cancer Association. Japanese classification of gastric carcinoma - 2nd English edition -. Gastric Cancer 1998; 1:10-24.

10. Nakajima T. Gastric cancer treatment guidelines in Japan. Gastric Cancer 2002:5:1-5.

11. Arai T, Esaki Y, Inoshita N, Sawabe M, Kasahara I, Kuroiwa K, et al. Pathologic characteristics of gastric cancer in the elderly: a retrospective study of 994 surgical patients. Gastric Cancer 2004; 7:154-9.

12. Naylor GM, Gotoda T, Dixon M, Shimoda T, Gatta L, Owen R, et al. Why does Japan have a high incidence of gastric cancer? Comparison of gastritis between UK and Japanese patients. Gut 2006;55:1545-52.

13. Asaka M, Kimura T, Kudo M, Takeda H, Mitani S, Miyazaki T, et al. Relationship of Helicobacter pylori to serum pepsinogens in an asymptomatic Japanese population. Gastroenterology 1992; 102:760-6.

14. Sasako M, McCulloch P, Kinoshita T, Maruyama K. New method to evaluate the therapeutic value of lymph node dissection for gastric cancer. Br J Surg 1995;82:346-51.

15. Shimada S, Yagi Y, Honmyo U, Shiomori K, Yoshida N, Ogawa M. Involvement of three or more lymph nodes predicts poor prognosis in submucosal gastric carcinoma. Gastric Cancer 2001; 4:54-9.

16. Nakamura K, Morisaki T, Sugitani A, Ogawa T, Uchiyama A, Kinukawa N, et al. An early gastric carcinoma treatment strategy based on analysis of lymph node metastasis. Cancer 1999;85: $1500-5$.

17. Seto Y, Shimoyama S, Kitayama J, Mafune K, Kaminishi M, Aikou T, et al. Lymph node metastasis and preoperative diagnosis of depth of invasion in early gastric cancer. Gastric Cancer 2001;4:34-8.

18. Yoshikawa T, Tsuburaya A, Kobayashi O, Sairenji M, Motohashi $\mathrm{H}$, Noguchi Y. Is D2 lymph node dissection necessary for early gastric cancer? Ann Surg Oncol 2002;9:401-5.

19. Popiela T, Kulig J, Kolodziejczyk P, Sierzega M. Long-term results of surgery for early gastric cancer. Br J Surg 2002;89: 1035-42. 